\title{
A Robotic Training System for Studies of Post-SCI Stand Rehabilitation*
}

\author{
Yongqiang Liang, Lance L. Cai, and Joel W. Burdick \\ Departments of Mechanical Engineering, Bioengineering \\ California Institute of Technology \\ 1200 E. California Blvd., Pasadena, CA 91125, U.S.A. \\ jwb@robotics.caltech.edu
}

\author{
V. Reggie Edgerton \\ Department of Neuroscience \\ University of California, Los Angeles \\ Los Angeles, CA 90095, U.S.A. \\ vre@ucla.edu
}

\begin{abstract}
This paper describes a robotic training system designed to rehabilitate the standing ability of mice after spinal cord injury(SCI). The system is composed of a 6 Degree-of-Freedom (DOF) parallel mechanism, an active weight support system, and other measuring equipments which can monitor the response of the mouse. Preliminary experiments showed that the mouse could generate a certain degree of weight-support stand response during the training.
\end{abstract}

Index Terms - Stand rehabilitation, parallel manipulator

\section{INTRODUCTION}

In the United States, an estimated 250,000 people live with Spinal Cord Injury (SCI), and an additional 11,000 new cases occur each year [8]. After SCI, the spinal cord circuity undergoes extensive change and the locomotion ability of the patients is lost or severely degraded ([7]). Such injuries lead to physical, emotional and financial loss to the injured people and their families.

The potential for rehabilitation after SCI has been an active research field for about 30 years ([1], [2]). For example, researchers developed motor prostheses to restore function movement of paralyzed limbs using Functional Neuromuscular Stimulus (FNS)([4], [5], [6]) or studied the issue via computer simulation ([9], [10]). Accumulated knowledge from these experiments shows the potential to harness the neural circuits and reflex pathways in the spinal cord to restore locomotion after SCI. These circuits and pathways are responsible for basic locomotion patterns and reflexes ([19]) and remain anatomically intact after SCI. However, the pathways to and from the supraspinal center are damaged and therefore their normal functions are changed ([7]).

One significant character of the spinal neural circuits is that they have use-dependent plasticity. If successfully exploited, this plasticity can be used to partially or fully restore the locomotion ability ([7]). There has been a growing awareness that in adult animal subjects, a high level of locomotion ability, such as weight-bearing stepping and standing, can be achieved following a complete SCI through training ([2], [3], [17], [18]) although the

\footnotetext{
* This work is partially supported by the Christopher Reeve Paralysis Foundation (\#VEC 2002-2), the National Institutes of Child Health and Humane Development and the National Institute of Neural Disorders and Stroke(1 R01 HD044830-01).
}

underlying physiological mechanism is still unclear yet. For example, after training, adult cats can regain weightbearing stepping and standing ability after complete SCI ([3], [12], [13], [20]). Rodents and other animals ([3]) can also achieve certain amount of locomotor ability after SCI. Pharmacological administration has also been employed together with training([3], [15], [14]), which have been shown to be able to enhance the effect of rehabilitative training. The human spinal cord has fair amount of plasticity, too. People with complete or incomplete SCI regained their locomotor independence in different levels ([24], [25]).

All these results show that the lower spinal cord below the lesion point has the ability to interpret afferent sensory information during standing or stepping. With appropriate afferent information of a particular motor task provided, the spinal cord can relearn to perform basic locomotion tasks even in the absence of supraspinal input.

Weight-bearing standing is an important functional for daily life. Paralyzed people would love to restore their weight-bearing stand ability so that they could carry out some daily activities such as washing dishes in their kitchen or standing in a social situation. Also, standing appears to involve a less complex neuromuscular model than stepping. In both senses, stand rehabilitation is an issue worthy to tackle.

Robotic devices have become more and more common in SCI rehabilitation. For example, the Locomat ${ }^{T M}$ is a commercially available robot for the rehabilitation of SCI and stroke patients. Robots can provide training consistency that can not be matched by therapists. And they can significantly reduce human effort during rehabilitative training. Also at the same time, robotic devices can provide precise quantification of the performance of the subjects after training. Being controlled by computer, the training program can be easily modified to suit different subjects. The training can also be adapted to the patient's progress. Thus, finding an effective robotic training paradigm has attracted the interest of researchers ([28], [29]). Bearing this in mind, we developed this robotic training system for standing rehabilitation in adult spinal mice. The mouse is a good model because of the availability of many strains of transgenic mice that enable detailed study of the pharmacology of locomotion. Also, detailed animal model 
study is preferred before the translations to human subjects ([11]).

The rest of the paper is arranged as follows. Section II discusses the motivations behind the design of the system. Section III describes the different parts of the system. Section IV describes some preliminary experiments. Section V concludes the paper and discusses the future experimental plan.

\section{DESIGN MOTIVATION}

As mentioned in the Introduction section, stand rehabilitation is potentially of great practical significance. Stand rehabilitative training has been carried out on spinal cats ([20]) and rats ([21]). Cutaneous inputs from the hind paw have been shown to be important in the locomotion control in spinal and intact cats ([26], [27]). In standing training, cutaneous inputs were also used as the stimuli to trigger the standing behavior. These prior studies show the need to provide both sensory stimuli and measurement of leg of the subject.

In [21], the Phantom ${ }^{T M}$ robot (which is a 3-DOF robotic arm) was used. The hindpaws of the mouse were attached to the end of the robotic arm so that perturbation can be applied to the hindlimbs. However, the contact between the hindpaws and the robot is not quite similar to the actual foot-ground contact. And therefore the cutaneous stimuli are not natural. On the other hand, due to the smaller body size of mouse (body length of 2 inches), it's hard to find a commercially available yet versatile enough device to train and test the spinal mice. Thus, we built a parallel manipulator with a 6-DOF plate end effector, which theoretically is able to generate any trajectory in it's workspace (for our platform, it's approximately a 2in cube). With the hindpaws of the mice on the moving plate and the forepaws supported by a fixed plate, we can stimulate the mice with different kinds of disturbances, such as vertical up and down, side-to-side translation, tilt, etc. so as to provide the spinal cord with load and stretch stimuli during standing.

After the complete spinal cord transection, mice typically can not support their body weight at all. Body weight support is needed to assist the animal to initiate a standing posture. How much weight the subject can bear by itself is an obvious measure of the training effect. With the performance of the subject changing, we wish to adjust the level of weight support adaptively. With this in mind, our system can actively adjust the amount of weight support under computer control. With the feedback from the animal's performance, an adaptive weight support schedule can also be applied during the training process.

The response of the mouse, including the joint angles, EMG signals of major muscle groups, and weight supported by the mouse (or ground reaction force), are recorded by the video tracking system, EMG electrodes, and load cells.

In the following section, details of the system design are given.

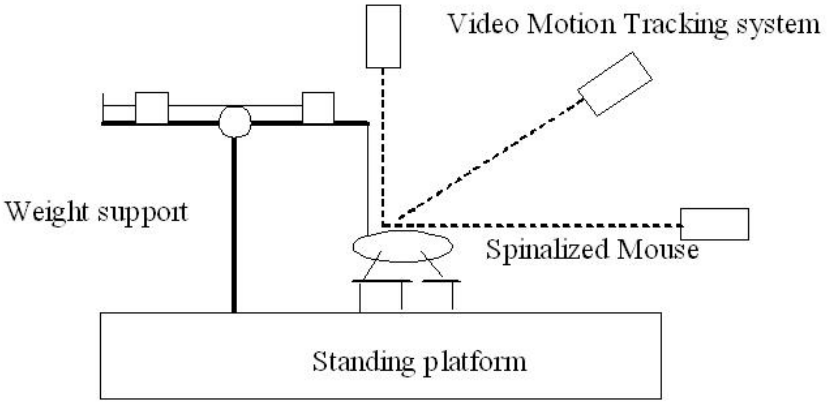

Fig. 1. The schematic of the robotic training system

\section{THE SYSTEM}

\section{A. System overview}

Fig. 1 shows the schematic of our system. The whole system is composed of a standing platform, an active weight support system and measuring equipments including joint angle measurement and body motion tracking. Fig. 2 is a photograph of the actual system (in which the video motion tracking system is not included). In this photo, a mouse is sitting in the system with a cable from the weight support attached to its sacrum. During training, the hindpaws of the mouse are put on the moving plate of the platform and the forepaws are on a fixed support. In our preliminary experiments, two suture loops are implanted under the sacrum connection tissue of the mice. Weight support is provided by hooking the mice to the weight support system through the implanted suture loops (A wisely designed harness could be more convenient than implanting suture loops in each mouse). Then different stimuli can be given to the hindlimbs when the moving plate moves along a prescribed trajectory. The active support system provides a certain level of weight support during the training. The motion tracking system can monitor the movement of a marker attached to the sacrum of the mouse. Together with the joint angles measured by fine optical fiber angle sensors, the animal's kinematic response to stimuli can be determined. The mouse's head is covered with a "hood" to keep out environmental distractions.

\section{B. Standing Platform}

A six Degree-of-Freedom (DOF) platform serves as the main training device. The platform is a parallel manipulator driven by four robotic linkages. The kinematic configuration of the device is shown in Fig. 3. The platform is modelled after the "NINJA" parallel manipulator in [16] because it has a compact configuration and potentially high moving speed and acceleration.

In the mechanism, each linkage has two active revolute joints and one passive revolute joint which is perpendicular with the two active ones. Parallelogram linkages are used here for the arrangement of the motors, as can be seen in 


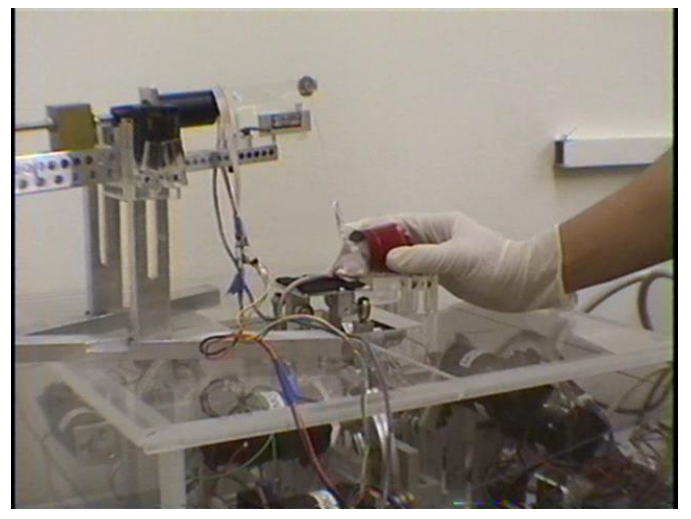

Fig. 2. The robotic training system with a mouse. The motion tracking system is not displayed in this photo

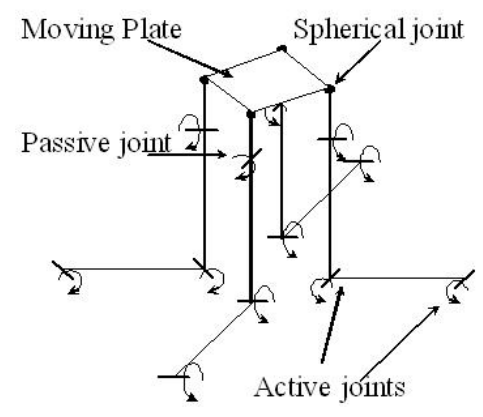

Fig. 3. Schematic configuration of the 6-DOF platform

the linkage model in Fig. 4. The platform is actually a redundant system since 3 of the four linkages are enough for generating the 6-DOF motion of the plate. But for the sake of mechanical balancing and the simplicity of trajectory planning, four identical linkages arranged symmetrically are used to drive the platform (Fig. 3). A plate is attached via spherical joints to the top of the four linkages.

To achieve high performance velocity and acceleration, the friction resistance between the mechanical parts should be minimized. For this purpose, 8 DC Motors drive the linkages through cable driven speed reducers (Fig. 5). The cables activate through tension so that there is almost zero friction as oppose to normal dog-tooth gears, and they are also maintenance free, i.e. free of lubrication. The motors are controlled by an 8 -axis PCI Galil ${ }^{T M}$ Motion Control board (Galil ${ }^{T M}$ 1800). The joint trajectories are precalculated by solving the inverse kinematics along desired training trajectories.

Our platform has 6 DOFs to enable arbitrary training trajectories. This allows us to have the freedom to try different stimuli and test the training effect with different disturbances.

\section{Active weight support system}

The weight support system (Fig. 8) has a balance weight whose position can be adjusted through a screw drive,

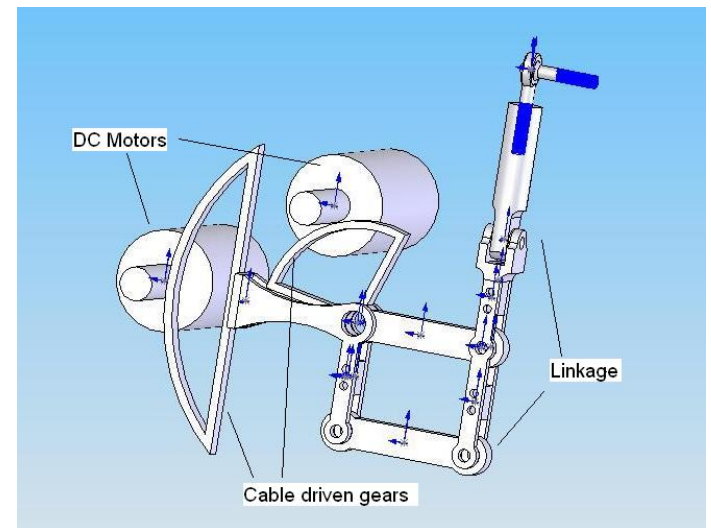

Fig. 4. The SolidWorks ${ }^{T M}$ model of a driving Linkage with two DC motors

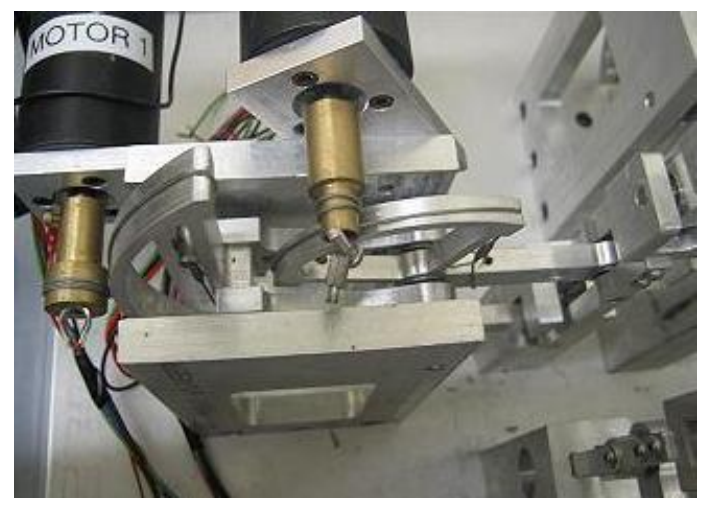

Fig. 5. A close view of the cable driven speed reducers

which is controlled by a DC motor. A load cell (GS050 form Transducer Techniques ${ }^{T M}$ with a measurement capacity of 50 grams) is used to monitor the tension in the cable which supports the mouse. An optical encoder is mounted on the pivot axis of the top lever to measure the tilt angle, from which we can infer the mouse posture. With the load cell readout as the feedback information, the motor is able to adjust the position of the balance weight and thus provide active body weight support to the mouse. The load cell can also provide additional information to quantify the weigh support ability of the mouse. The weight support system is controlled by a 4axis PCI Galil ${ }^{T M}$ Motion Control Board (Galil $\left.{ }^{T M} 1840\right)$. Currently, a feedback control based on the load cell reading is used to maintain a certain level of weight support. To prevent the weight support lever from turning beyond some certain angle (the mouse could be pulled away from the platform or collapse too much) the pivot angle from the optical encoder is monitored to keep the lever between the lower and upper angle boundaries.

\section{Performance measurement}

To monitor the response of the mouse during training, a $3 \mathrm{D}$ motion tracking system is built to track the motion of a marker attached to the sacrum point. Three non-interlaced CCD cameras (MC-CC-P60 60fps progressive scan "Cube Cam", resolution $659 \times 497$ pixels) and an multichannel 


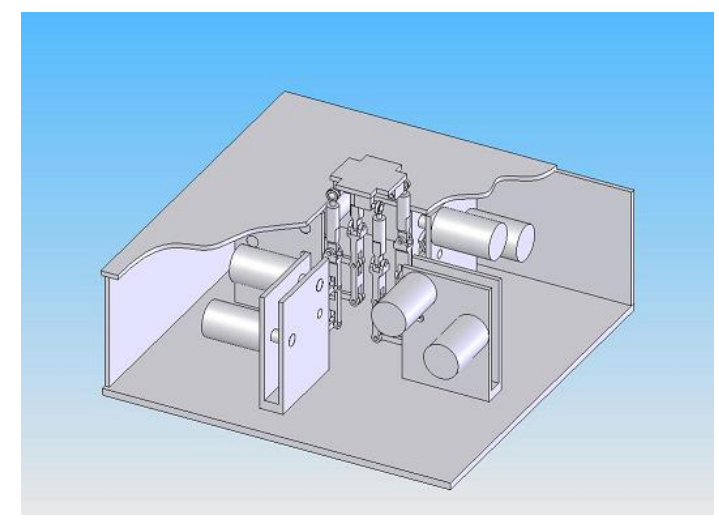

Fig. 6. The SolidWorks ${ }^{T M}$ model of platform

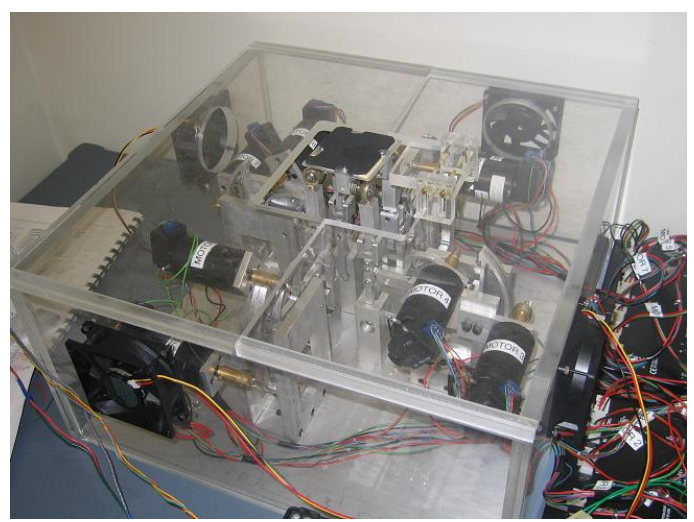

Fig. 7. The standing platform

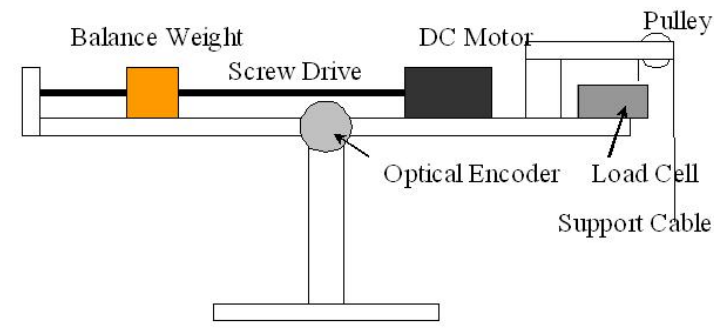

Fig. 8. The schematic of the active weight support system

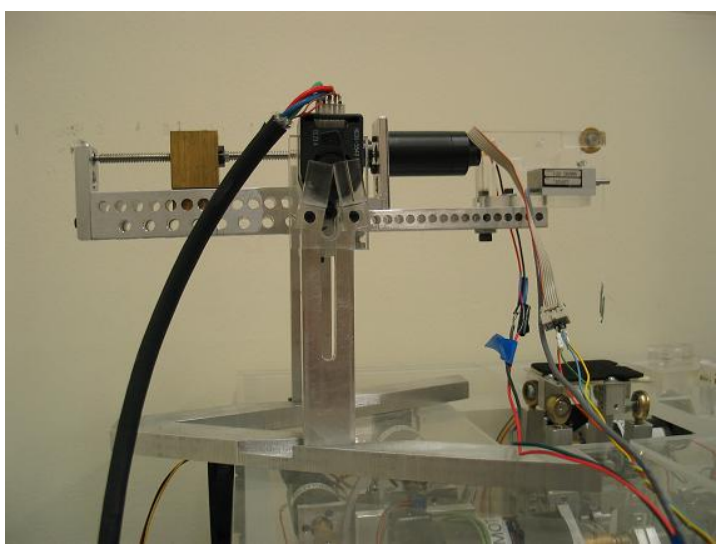

Fig. 9. The active weight support system frame grabber (PC2Vision ${ }^{T M}$ from Dalsa Coreco) are used to record the motion of the marker point at a frequency of $30 \mathrm{~Hz}$. Progressive scan can eliminate the motion blur. Real-time image processing programs can then provide the $3 \mathrm{D}$ coordinates of the marker point, which give us the base point of the hindlimbs. To measure the major joint angles we use S720 miniature Shape Sensor ${ }^{T M}$ s from Measurand. These are optical fiber angle sensors with diameter less than $1.5 \mathrm{~mm}$, which are fine enough to be attached to the hindlimbs of the mouse and have a resolution of 0.5 degree.

Ground reaction force sensors are mounted on the moving plate of the platform. So the force applied to the hindpaws during the experiment can be recorded.

Optionally, acute EMG electrodes can be implanted in the major extensor and flexor groups during experiments. By comparing the EMG behavior, hindlimb kinematics, ground reaction force generated by different stimuli, we hope we can get some insight into the optimal stimulation for standing rehabilitation and further reveal the mechanism underlying the full-weight-bearing stand.

\section{PRELIMINARY EXPERIMENTS}

The integrated system can test different training strategies. It has the potential ability to provide adaptive training based on the current performance of the animal.

\section{A. Subject}

Adult Swiss-Webster mice (body weight around $25 \mathrm{~g}$ ) obtained from Charles River Laboratories (Wilmington, MA) were used. A complete mid-thoracic spinal cord transection was performed at 45 days of age.

\section{B. Experiments}

To date, simple pilot studies have been carried out to test the system's capacity. Sinusoidal and trapezoidal (duty cycle of 30\%) vertical perturbations (Fig. 10) with magnitude of $2 \mathrm{~mm}$ and frequencies of 1,2 and $3 \mathrm{~Hz}$ were given to the mice with or without quipazine (a 5HT agonist which shown to be able to enhance the training effect in stepping, [14]). Quipazine was administrated by a dosage of $0.5 \mu \mathrm{g} / \mathrm{g}$ body weight. Results showed that among the stimuli we tried, the $2 \mathrm{~Hz}$ trapezoid perturbation triggered the most response of the mice, which, as evidenced by an increased angle between the paw and the support surface after training. Fig. 11 shows a comparison before and after training. Note the difference between the angle of the ankle. When quipazine was administrated, the mice had a stronger and easier response to the stimuli.

After training, the increased paw angle is closer to normal postures and the hindlimbs support more weight. Therefore, the animal demonstrates improved standing ability.

\section{CONCLUSION AND FUtURE PLAN}

We developed a robotic training system for the studies of post-SCI stand rehabilitation of mice. The preliminary studies demonstrated the potential of our system and the feasibility to carry out the rehabilitation study for mice. We 

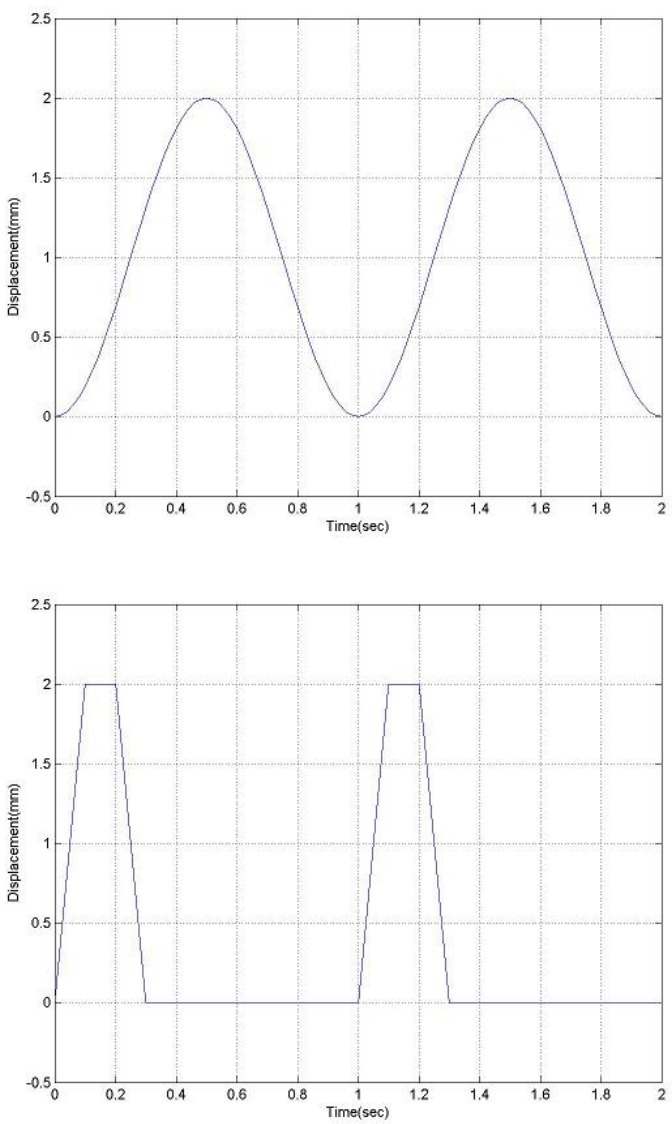

Fig. 10. Examples of the perturbation signals

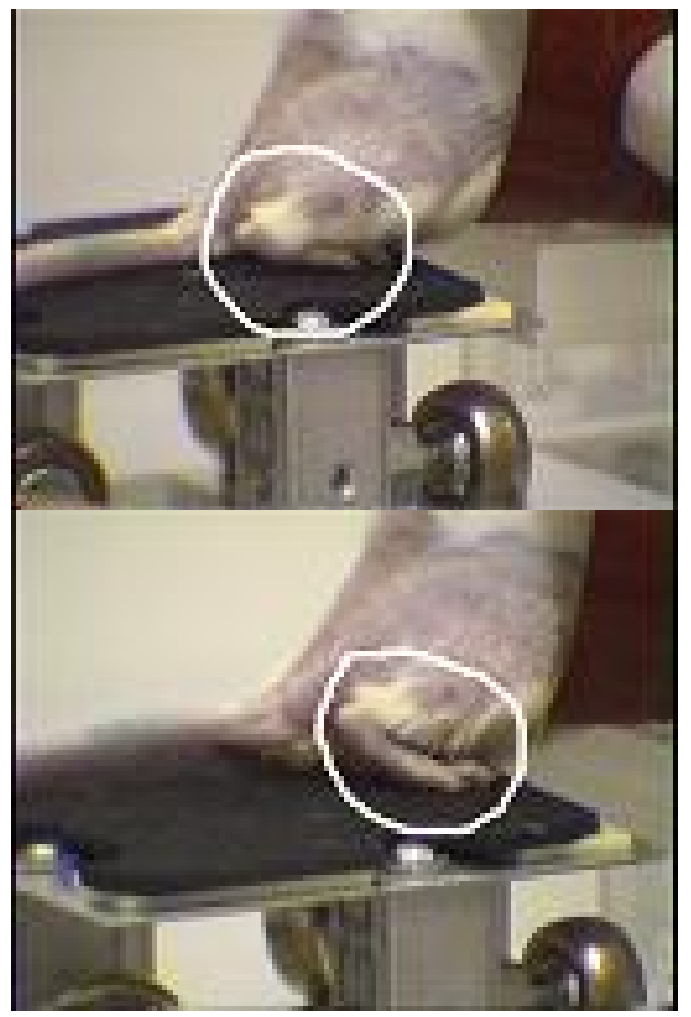

Fig. 11. The snapshots of the hindlimb before and after training are currently planning for more sophisticated experiments in the near future that will use the active feedback of mouse posture to enhance the training effect.

\section{ACKNOWLEDGMENT}

This work is partially supported by the Christopher Reeve Paralysis Foundation (\#VEC 2002-2), the National Institutes of Child Health and Humane Development and the National Institute of Neural Disorders and Stroke(1 R01 HD044830-01)

\section{REFERENCES}

[1] H. Barbeau, D. A. McCrea, M. J. O’Donovan, S. Rossignol, W. M. Grill, and M. A. Lemay, "Tapping into Spinal Circuits to Restore Motor Function", Brain Research Reviews 30: 27-51, 1999

[2] K. Fouad and K. Pearson, "Restoring Walking after Spinal Cord Injury", Prog. Neirobio. 73: 107-126, 2004

[3] S. Rossignol, "Locomotion and it's recovery after spinal injury", Curr. Opin. Neurobio. 10: 708-716, 2000

[4] W. T. Liberson, H. J. Holmoquest, D. Scott and A. Dow, "Functional Electrotherapy: Stimulation of the Peroneal Nerve Synchronized with the Swing Phase of the Gait in Hemiplegic Patients", Arch. Phys. Med. Rehabil. 42: 101-105, 1961

[5] M. R. Carhart, J. He, R. Herman, S. S'Luzansky, and W. T. Willis, "Epidural Spinal-Cord Stimulation Failitates Recovery of Functional Walking following Incomplete Spinal-Cord Injury”, IEEE Trans. Neur. Sys. Rehabil. Eng. 12(1): 32-42, 2004

[6] G. M. Lyons, T. Sinkær, J. H. Burridge, and D. J. Wilcox, "A Review of Portable FES-Based Neural Orthoses for the Correction of Drop Foot”, IEEE Trans. Neur. Sys. Rehabil. Eng. 10(4): 260-279, 2002

[7] V. Dietz and R. Müller, "Degradation of Neuronal Function following a Spinal Cord Injury: Mechanisms and Countermeasures", Brain 127: 2221-2231, 2004

[8] Spinal Cord Injury Information Network, available online at http://images.main.uab.edu/spinalcord/ pdffiles/factsfig.pdf (accessed on Oct. 6, 2005)

[9] G. Khang and F. E. Zajac, "Paraplegic Standing Controlled by Functional Neuromuscular Stimulation: Part I - Computer Model and Control System Design", IEEE Trans. Biomed. Eng. 36(9): 873884,1989

[10] G. Khang and F. E. Zajac, "Paraplegic Standing Controlled by Functional Neuromuscular Stimulation: Part II - Computer Simulation Studies", IEEE Trans. Biomed. Eng. 36(9): 885-894, 1989

[11] M. L. Sipski, "From the Bench to the Body: Key Issues with Research Aimed at a Cure for SCI", J. Rehabil. Res. and Develop. 40(4): 1-8, 2003

[12] R. D. de Leon, J. A. Hodgson, R. R. Roy, and V. R. Edgerton, "Locomotor Capacity Attributable to Step Training versus Spontaneous Recovery Following Spinalization in Cats", J. Neurophysiol. 79: 1329-1340, 1998

[13] R. G. Lovely, R. G. Gregor, R. R. Roy, and V. R. Edgerton, "Effects of Training on the Recovery of Full-Weight-Bearing Stepping in the Adult Spinal Cat", Exp. Neurol. 92: 421-435, 1986

[14] A. J. Fong, V. R. Edgerton, L. L. Cai, C. K. Otoshi, H. Zhong, R. R. Roy, D. J. Reinkensmeyer, and J. W. Burdick, "Effects of Quipazine and Robotic Training on Spinal Mice", Soc. Neurosci. Abstr. 498.20, 2003

[15] C. Chau, H. Barbeau, and S. Rossignol, "Early Locomotro Traing with Clonidine in Spinal Cats", J. Neurophysiol. 79: 392-409, 1998

[16] K. Nagai, M. Matsumoto, K. Kimura and B. Masuhara, "Development of Parallel Manipulator 'NINJA' with Ultra-HighAccelaration", Proc. 2003 IEEE Intern. Conf. Rob. and Auto., pp. 1117-1184, 2003.

[17] V. R. Edgerton, R. D. de Leon, S. J. Harkema, J. A. Hodgson, N. London, et al. "Retraining the Injured Spinal Cord", J. Physiol. 533: 15-22, 2001

[18] V. R. Edgerton, N. J. Tillakaratne, A. J. Bigbee, R. D. de Leon, and R. R. Roy, "Plasticity of The Spinal Neural Circuitry after Injury", Annu. Rev. Neurosci. 27: 145-167, 2004.

[19] E. Marder and R. L. Calabrese, "Principles of Rhythmic Motor Pattern Generation”, Physiol. Rev. 76(3): 687-717, 1996 
[20] R. D. de Leon, J. A. Hodgson, R. R. Roy and V. R. Edgerton, "Full Weight-Bearing Hindlimb Standing Following Stand Training in the Adult Spinal Cat", J. Neurophysiol. 80: 83-91, 1998.

[21] A. J. Bigbee, N.J.K. Tillakaratne, J. Hodgson, R. R. Roy, H. Zhong R. Shah, A. Olivas, A. J. Tobin, and V. R. Edgerton, "Quantitative Analysis of Standing Ability Following Stand Training in Rats after Spinal Cord Transection", Soc. Neurosci. Abstr. 857.3, 2003

[22] C. A. Pratt, J. Fund, and J. M. Macpherson, "Stance Control in the Chronic Spinal Cat", J. Neurophysiol. 70: 1981-1985, 1994

[23] J. M. Macpherson and J. Fung, "Weight Support and Balance During Perturbed Stance in the Chronic Spinal Cat", J. Neurophysiol. 82: 3066-3081, 1999.

[24] S. J. Harkema, S. L. Hurley, U. K. Patel, P. S. Requejo, B. H. Dobkin, and V. R. Edgerton, "Human Lumbosacral Spinal Cord Interprets Loading during Stepping", J. Neurophysiol. 77: 797-811, 1997

[25] C. K. Ferreria, S. J. Harkema, and J. Beres-Jones, "Neural Reorganization of the Functionally Isolated Human Spinal Cord Occurs after Stand Training", Web Post, http: / / www . harkema . ucla. edu/standTrainingPoster2003.html

[26] L. J. G. Bouyer and S. Rossignol, "Contribution of Cutaneous Inputs from the Hindpaw to the Control of Locomotion. I. Intact Cats", $J$. Neurophysiol. 90: 3625-3639, 2003

[27] L. J. G. Bouyer and S. Rossignol, "Contribution of Cutaneous Inputs from the Hindpaw to the Control of Locomotion. II. Spinal Cats", J. Neurophysiol. 90: 3640-3653, 2003

[28] S. Jezernik, R. Schärer, G. Golombo, and M. Morari, "Adaptive Robotic Rehabilitation of Locomotion: a Clinical Study in Spinally Injured Individuals", Spinal Cord 41: 657-666, 2003

[29] S. Jezernik, R. Schärer, G. Golombo, and M. Morari, "Automatic Gait-Pattern Adaptation Algorithms for Rehabilitation with a 4-DOF Robotic Orthosis", IEEE. Trans. Rob. Auto. 20(3):574-582, 2004

[30] L. L. Cai, A. J. Fong, C. K. Otoshi, Y. Q. Liang, J. G. Cham, H. Zhong, R. R. Roy, V. R. Edgerton, J. W. Burdick, "Effects of Consistency vs. Variability in Robotically Controlled Training of Stepping in Adult Spinal Mice", Proc. 2005 IEEE Intern. Conf. Rehabil. Rob. 575-579, 2005, Chicago, IL, USA 